Proceeding Series of the Brazilian Society of Computational and Applied Mathematics

\title{
Estimativas para $n$-Larguras de Kolmogorov de Conjuntos de Funções Finita e Infinitamente Diferenciáveis sobre o Toro
}

\author{
Régis Leandro Braguim Stábile ${ }^{1}$ \\ IFSP - Campus Birigui, Birigui, SP \\ Sergio Antonio Tozoni ${ }^{2}$ \\ Departamento de Matemática, IMECC, UNICAMP, Campinas, SP
}

\begin{abstract}
Resumo. A teoria de $n$-larguras foi introduzida por Kolmogorov na década de 1930. Desde então, muitos trabalhos têm visado obter estimativas assintóticas para $n$-larguras de diferentes classes de conjuntos. Neste trabalho, investigamos $n$-larguras de Kolmogorov de operadores multiplicadores associados a conjuntos de funções finitamente e infinitamente diferenciáveis sobre o toro. Em particular, demonstramos que as estimativas obtidas são assonticamente exatas em termos de ordem em diversas situações.
\end{abstract}

Palavras-chave. n-larguras, Kolmogorov, toro, operadores multiplicadores.

\section{Introdução}

Seja $A$ um subconjunto compacto e centralmente simétrico de um espaço de Banach $X$. Definimos a $n$-largura de Kolmogorov de $A$ em $X$ por

$$
d_{n}(A, X)=\inf _{X_{n}} \sup _{x \in A} \inf _{y \in X_{n}}\|x-y\|_{X},
$$

onde o ínfimo é tomado sobre todos os espaços $n$-dimensionais $X_{n}$ de $\mathrm{X}$, tal valor nos diz o quão bem podemos aproximar o conjunto $A$ por subespaços finito dimensionais de $X$. Se $Y$ é um outro espaço de Banach e $T: X \longrightarrow Y$ um operador limitado, definimos a $n$ largura de Kolmogorov de $T$ por $d_{n}(T)=d_{n}\left(T\left(B_{X}\right), Y\right)$, onde $B_{X}$ denota a bola unitária fechada do espaço $X$.

Seja $\Lambda=\left\{\lambda_{\mathbf{k}}\right\}_{\mathbf{k} \in \mathbb{Z}^{d}}, \lambda_{\mathbf{k}} \in \mathbb{R}$, e sejam $1 \leq p, q \leq \infty$. Se para todo $\varphi \in L^{p}\left(\mathbb{T}^{d}\right)$ existe uma função $f=\Lambda \varphi \in L^{q}\left(\mathbb{T}^{d}\right)$ com expansão formal em série de Fourier dada por

$$
f \sim \sum_{\mathbf{k} \in \mathbb{Z}^{d}} \lambda_{\mathbf{k}} \widehat{f}(\mathbf{k}) e^{i \mathbf{k} \cdot \mathbf{x}},
$$

tal que $\|\Lambda\|_{p, q}=\sup \left\{\|\Lambda \varphi\|_{q}: \varphi \in U_{p}\right\}<\infty$, dizemos que $\Lambda$ é um operador multiplicador limitado de $L^{p}$ em $L^{q}$, com norma $\|\Lambda\|_{p, q}$, onde $U_{p}$ denota a bola unitária fechada do espaço $L^{p}\left(\mathbb{T}^{d}\right)$. Consideraremos operadores multiplicadores $\Lambda=\left\{\lambda_{\mathbf{k}}\right\}_{\mathbf{k} \in \mathbb{Z}^{d}}$, onde $\lambda_{\mathbf{k}}=\lambda(|\mathbf{k}|)$ para uma função real $\lambda$ definida sobre $[0, \infty)$ e $|\cdot|$ denota a norma euclidiana.

\footnotetext{
${ }^{1}$ registabile@ifsp.edu.br

2 tozoni@ime.unicamp.br
} 


\section{Resultados}

Se $\Lambda^{(1)}=\left\{\lambda_{\mathbf{k}}\right\}_{\mathbf{k} \in \mathbb{Z}^{d}}$, onde a função $\lambda:[0, \infty) \rightarrow \mathbb{R}$ é definida por $\lambda(t)=t^{-\gamma}(\ln t)^{-\xi}, t>$ 1 e $\lambda(t)=0$ para $0 \leq t \leq 1, \gamma, \xi \in \mathbb{R}, \gamma>d / 2, \xi \geq 0$, temos que $\Lambda^{(1)} U_{p}$ são conjuntos de funções finitamente diferenciáveis sobre $\mathbb{T}^{d}$, em particular, se $\xi=0$ então $\Lambda^{(1)} U_{p}$ são classes de Sobolev.

Se $\Lambda^{(2)}=\left\{\lambda_{\mathbf{k}}\right\}_{\mathbf{k} \in \mathbb{Z}^{d}}$, onde a função $\lambda:[0, \infty) \rightarrow \mathbb{R}$ é definida por $\lambda(t)=e^{-\gamma t^{r}}, \gamma, r>0$, temos que $\Lambda^{(2)} U_{p}$ são conjuntos de funções infinitamente diferenciáveis (analíticas para $r=1)$.

Para os resultados seguintes, usaremos as notações

$$
\vartheta_{n}=\left\{\begin{array}{cc}
1, & 1 \leq p \leq 2,1<q \leq 2 \\
1, & 2 \leq p<\infty, 2 \leq q \leq \infty \\
1, & 1 \leq p \leq 2 \leq q \leq \infty, \\
(\ln n)^{-1 / 2}, & 1 \leq p \leq 2, q=1 \\
(\ln n)^{-1 / 2}, & p=\infty, 2 \leq q \leq \infty
\end{array} \quad, \quad(a)_{+}= \begin{cases}a, & a>0 \\
0, & a \leq 0\end{cases}\right.
$$

$a_{n} \gg b_{n}$ e $a_{n} \ll b_{n}$, se existirem constantes positivas $C_{1}$ e $C_{2}$ tais que $a_{n} \geq C_{1} b_{n}$ e $a_{n} \leq C_{2} b_{n}$, respectivamente, para todo $n \in \mathbb{N}$. Se tivermos $a_{n} \gg b_{n}$ e $a_{n} \ll b_{n}$, escreveremos $a_{n} \asymp b_{n}$.

Teorema 2.1. Seja $\Lambda^{(1)}$ o operador multiplicador definido acima. Então para $1 \leq p \leq \infty$, $2 \leq q \leq \infty$ e para todo $n \in \mathbb{N}$, temos

$$
d_{n}\left(\Lambda^{(1)} U_{p} ; L^{q}\right) \ll n^{-\gamma / d+(1 / p-1 / 2)_{+}}(\ln n)^{-\xi} \begin{cases}q^{1 / 2}, & q<\infty, \\ (\ln n)^{1 / 2}, & q=\infty,\end{cases}
$$

e para $1 \leq p, q \leq \infty$, temos

$$
d_{n}\left(\Lambda^{(1)} U_{p}, L^{q}\right) \gg n^{-\gamma / d}(\ln n)^{-\xi} \vartheta_{n} .
$$

Teorema 2.2. Seja $\Lambda^{(2)}$ o operador multiplicador definido acima e $\mathcal{R}=\gamma\left(d \Gamma(d / 2) / 2 \pi^{d / 2}\right)^{r / d}$. Então para todo $n \in \mathbb{N}$ e $0<r \leq 1$

$d_{n}\left(\Lambda^{(2)} U_{p}, L^{q}\right) \gg e^{-\mathcal{R} n^{r / d}} \vartheta_{n}, \quad 1 \leq p, q \leq \infty$,

$d_{n}\left(\Lambda^{(2)} U_{p}, L^{q}\right) \ll e^{-\mathcal{R} n^{r / d}} n^{(1-r / d)(1 / p-1 / 2)_{+}}\left\{\begin{array}{ll}1, & 2 \leq q<\infty, \\ (\ln n)^{1 / 2}, & q=\infty,\end{array} \quad 1 \leq p \leq \infty, 2 \leq q \leq \infty\right.$.

Corolário 2.1. Para $2 \leq p, q<\infty$ e $0<r \leq 1$, temos que

$$
d_{n}\left(\Lambda^{(1)} U_{p}, L^{q}\right) \asymp n^{-\gamma / d}(\ln n)^{-\xi} \quad \text { e } \quad d_{n}\left(\Lambda^{(2)} U_{p}, L^{q}\right) \asymp e^{-\mathcal{R} n^{r / d}} .
$$

\section{Referências}

[1] L. Grafakos, Classical Fourier Analysis, Springer, 2 Ed., (2008).

[2] A. Kushpel, R. L. B. Stabile e S. A. Tozoni, Estimates for n-widths of sets of smooth function on the torus $T^{d}$, Journal of Approximation Theory, vol. 183, 45-71, (2014).

[3] A. Pinkus, n-Widths in Approximation Theory, Springer Verlag, (1985). 